\title{
Modeling and Analysis of Vertical Noise Coupling in TSV-Based 3D Mixed-Signal Integration
}

\author{
Shiwei Wang ${ }^{1}$, Yingtao Ding ${ }^{*}$, Zhiming Chen ${ }^{1}$, Huanyu $\mathrm{He}^{2}$ and Jian-Qiang $\mathrm{Lu}^{2}$ \\ 1. School of Information and Electronics, Beijing Institute of Technology, Beijing, 100081, China, \\ 2. Department of Electrical, Computer, and Systems Engineering, Rensselaer Polytechnic Institute, Troy, NY \\ 12180 USA \\ *Corresponding author: ytd@bit.edu.cn
}

\begin{abstract}
This paper reports on modeling and analysis of vertical noise coupling between adjacent chips in TSVs-based 3D mixed-signal integration. The coupling between clock channel in digital IC and channel routing wires (CRWs) in analog IC is studied in both frequency domain and time domain. According to physical architecture, a broadband equivalent circuit model of vertical noise coupling is proposed. The comparison of S parameters from full-wave electromagnetic (EM) simulation and circuit model shows a good agreement. The coupling mechanism is discussed from transfer impedance between clock channel and CRWs. The impacts of design variables (such as geometry dimension, 3D stacking process, layout floorplan) on noise coupling are investigated. Four approaches are proposed to reduce the vertical noise coupling and compared with the baseline model. Finally, the time domain analysis shows that the rising/falling time determines peak-to-peak noise voltage and the far-end exhibits larger noise than the near-end.
\end{abstract}

Keywords: Channel routing wire; Clock channel; Equivalent circuit model; Mixed-signal; Through silicon vias (TSVs); Vertical noise coupling.

\section{Introduction}

Motivated by the requirements of high speed, high bandwidth and high power efficient electronic systems in modern society, three-dimensional (3D) integration technology based on vertical interconnects that penetrate through silicon strata was developed [1]-[4]. It is promising to be applied into fields like memory [5]-[7], image sensors[8]-[10] and microprocessors[11][12] for it involves lower interconnect latency, lower power consumption, smaller form factor and easier for heterogeneous integration between different technologies.

In 3D mixed-signal integration systems, analog circuits, digital circuits and sensors are usually vertically stacked. Kiyoyama et al [13] has proposed a 3D stacked image processing system, in which, a parallel analog digital convertor (ADC) with hierarchical correlated double sampling technique is vertically stacked with image processing circuit by TSVs. Liu et al [14] reports the design of a TSV based three-layer stacked 12-bit successive approximation register (SAR) ADC, where the analog parts of ADC are stacked on top of digital parts. Since analog circuits such as readout integrated circuits (ROIC) and ADC are sensitive to the switching noise from digital circuits, it is essential to evaluate the characteristics of vertical coupling behavior between analog circuits and digital circuits in mixed-signal 3D integration systems, especially for state-of-the-art 3D integration scheme, in which, silicon substrates are thinned down to about $50 \mu \mathrm{m}$ or less and vertical noise coupling between heterogeneous chips becomes more severe. All in all, by applying TSV technology into 3D mixed-signal integration systems, relatively shorter signal path and reduced 
form factor can be obtained, but on the other hand, it raises concerns about vertical noise coupling from digital to analog because of the thin chip substrate and high switching frequency. Therefore, the demands of investigation about vertical noise coupling in TSV-based 3D mixed-signal integration will be urgent.

Previous works have shown that vertical noise coupling between adjacent chips are significant to performance degradation in 3D devices [15][16]. However, these studies have focused on the analysis of vertical crosstalk in 3D system in which the connections between adjacent chips are realized through wire bonding. In contrast, this work provides the detailed analysis of the vertical noise coupling in TSV-based 3D mixed-signal integration. By applying finite element methods (FEM), EM field is solved to capture parasitics and couplings. The corresponding broadband equivalent circuit model is proposed and compared with EM solution up to 40GHz. Moreover, the coupling mechanism and impacts of design variables are analyzed in detail, based on which four methods are proposed to mitigate the noise coupling. Furthermore, the transient noise transfer characteristics such as ringing effect and peak-to-peak noise voltage at both near-end and far-end are also investigated.

\section{Modeling}

\subsection{TSV-Based 3D Architecture for Mixed-Signal Integration}

In synchronous digital systems, clock signal is used as time reference for data processing, typically passes through the longest distance and works at the high speed [17]. The clock distribution is to deliver global clock across the chip to reach all of the clocked elements [18]. In analog or mixed-signal layout design, a technique that routing the wires alongside the individual circuit blocks is known as channel routing [19]. Fig.1 shows a baseline 3D structure employed to model the vertical noise coupling between the clock channel and channel routing wires (CRWs). The baseline architecture is constituted of a top analog chip and a bottom digital chip bonded together with benzocyclobutene (BCB). The clock channel is realized using the top metal layer (M9) embedded in inter-metal dielectric layer 2 (IMD2) of the bottom tier while the CRWs (labeled as ' $a$ ' and ' $b$ ') are made of M3 embedded in IMD1 of the top tier. The ground grids made of M8 and M9 in each tier are connected with six TSVs. The geometry and material parameters are listed in Table I, which are based on TSMC $90 \mathrm{~nm}$ CMOS technology. Clock_M9 and CRW_M3 represent the clock channel and CRWs, respectively. Ground_M9 and Ground_M8 connected by Via_M8_M9 form the ground grids. Substrate (T) and substrate (B) stand for the top and bottom substrate, respectively. The area of substrate $(600 \mu \mathrm{m} \times 600 \mu \mathrm{m})$ is chosen by considering the typical size of building block in mixed-signal circuit design. And the initial width, length, and space of metal wires are determined from common practice in mixed-signal circuit design, while the impact of geometry dimension on noise coupling is analyzed in section 3. The thicknesses of the top substrate, bottom substrate, and bonding material as well as the geometry of TSVs are chosen based on the realistic fabrication process of 3D integration.

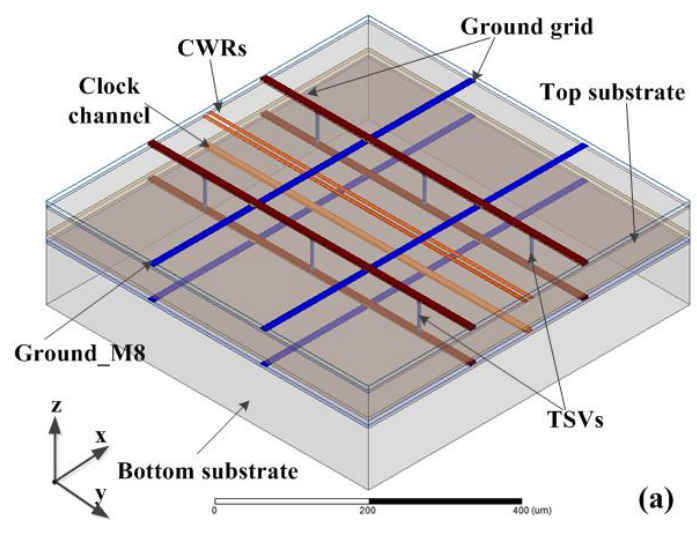

Fig. 1. (a) 3D view of baseline model with two stacked chips.

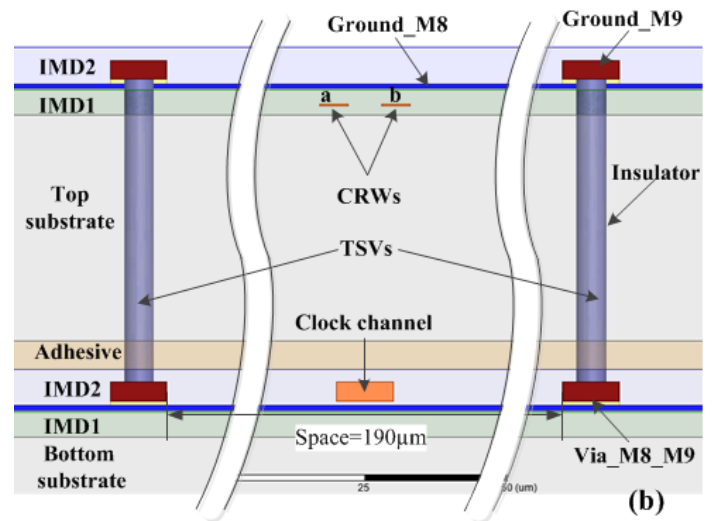

(b) Sideview of baseline model for simulation (not in scale). 
TABLE I

DIMENSION AND MATERIAL PARAMETER OF BASELINE MODEL

\begin{tabular}{ccccccc}
\hline \hline Name & $\begin{array}{c}\text { Width } \\
(\mu \mathrm{m})\end{array}$ & $\begin{array}{c}\text { Length } \\
(\mu \mathrm{m})\end{array}$ & $\begin{array}{c}\text { Thickness } \\
(\mu \mathrm{m})\end{array}$ & $\begin{array}{c}\text { Space } \\
(\mu \mathrm{m})\end{array}$ & $\begin{array}{c}\text { Relative } \\
\text { permittivity }\end{array}$ & $\begin{array}{c}\text { Conductivity } \\
(\mathrm{S} / \mathrm{m})\end{array}$ \\
\hline Clock_M9 & 10 & 600 & 3.4 & - & 1 & $5.88 \times 10^{7}$ \\
CRW_M3 & 5 & 600 & 0.31 & 6 & 1 & $4.48 \times 10^{7}$ \\
Ground_M9 & 10 & 600 & 3.4 & 190 & 1 & $5.88 \times 10^{7}$ \\
Ground_M8 & 10 & 600 & 0.85 & 190 & 1 & $5.35 \times 10^{7}$ \\
Via_M8_M9 & 10 & 10 & 0.75 & 190 & 1 & $1.49 \times 10^{7}$ \\
Substrate(T) & 600 & 600 & 40 & - & 11.9 & 10 \\
Substrate(B) & 600 & 600 & 100 & - & 11.9 & 10 \\
IMD1 & 600 & 600 & 4.5 & - & 2.9 & 0 \\
IMD2 & 600 & 600 & 7.5 & - & 4.2 & 0 \\
Bonding material & 600 & 600 & 5 & - & 2.6 & 0 \\
TSV & 5 (Diameter) & 53.6 & - & 195 & 1 & $5.88 \times 10^{7}$ \\
Insulator & - & 53.6 & 0.3 & 195 & 3.9 & 0 \\
\hline \hline
\end{tabular}

\subsection{EM Solution}

ANSYS's HFSS is employed as a 3D EM field solver to capture parasitics and simulate vertical noise coupling between the clock channel and CRWs in the baseline model. All ports are terminated with $50 \Omega$ and the ports allocation are shown in Fig. 2(a). Due to the symmetry of CRWs ' $a$ ' and ' $b$ ' with respect to the clock channel, the near-end crosstalk (NEXT) $S_{31}$ and far-end crosstalk (FEXT) $S_{41}$ are equal to $S_{51}$ and $S_{61}$, respectively. As shown in Fig. 2(b), the fluctuation of $S_{11}$ at high frequency indicates the self-resonation occurs between the parasitic capacitance and the self-inductance of clock channel. Besides, NEXT and FEXT increase with frequency until approaching the self-resonant frequency, and the crosstalk $\left(\mathrm{S}_{31}\right.$ and $\left.\mathrm{S}_{41}\right)$ is larger than $-30 \mathrm{~dB}$ above 3 $\mathrm{GHz}$, namely, a 1-V signal transmitted through the clock channel can generate more than $31 \mathrm{mV}$ noise at the CRWs, which may overwhelm the small analog signal and severely degrade the performance of the analog chip.
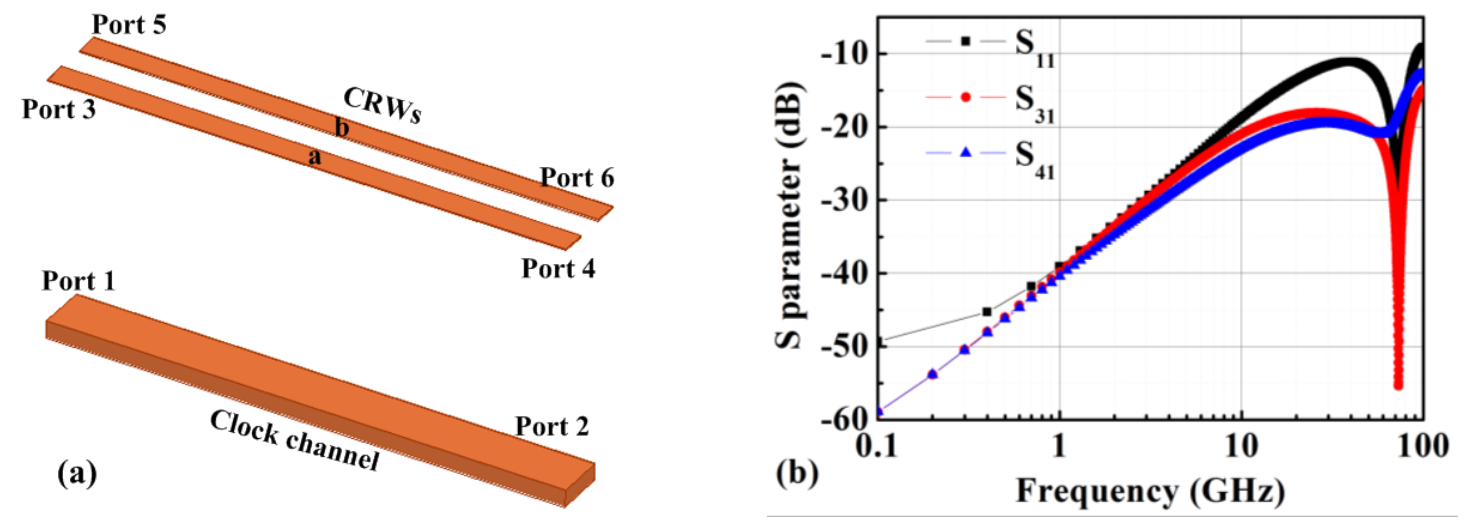

Fig. 2. (a) Ports allocation of the baseline model. (b) Simulated reflection coefficient $\left(\mathrm{S}_{11}\right)$, NEXT $\left(\mathrm{S}_{31}\right)$ and FEXT $\left(\mathrm{S}_{41}\right)$ from $0.1 \mathrm{GHz}$ to $100 \mathrm{GHz}$.

\subsection{Coupling Mechanism}

Capacitive coupling through parasitic capacitance and inductive coupling through mutual inductance are the two typical coupling paths between clock channel and CRWs. In order to understand the dominant coupling mechanism of the baseline model, transfer impedance is extracted from the EM solution, which represents the coupled noise voltage in the CRW induced by a unit current injected into the clock channel. As shown in Fig. 3(a), the transfer impedance increases linearly with frequency and exhibits a pure inductive characteristics from $0.1 \mathrm{GHz}$ to $15 \mathrm{GHz}$, where the capacitive coupling can be ignored. Fig. 3(b) depicts the mutual inductance derived from transfer impedance, which is constant with $0.16 \mathrm{nH}$ up to $15 \mathrm{GHz}$. This indicates that the magnetic fields generated by currents flowing through the clock channel and CRWs strongly couple with each other, which makes the inductive coupling become the dominant path. The capacitive coupling starts to play a role at frequency above $15 \mathrm{GHz}$ because the transfer impedance increases faster than linear with frequency, in other words, the equivalent mutual inductance increases with frequency, as can be seen from Fig. 3(b). The capacitive coupling dominates beyond $40 \mathrm{GHz}$ where the transfer impedance decreases with frequency and the equivalent mutual inductance becomes 

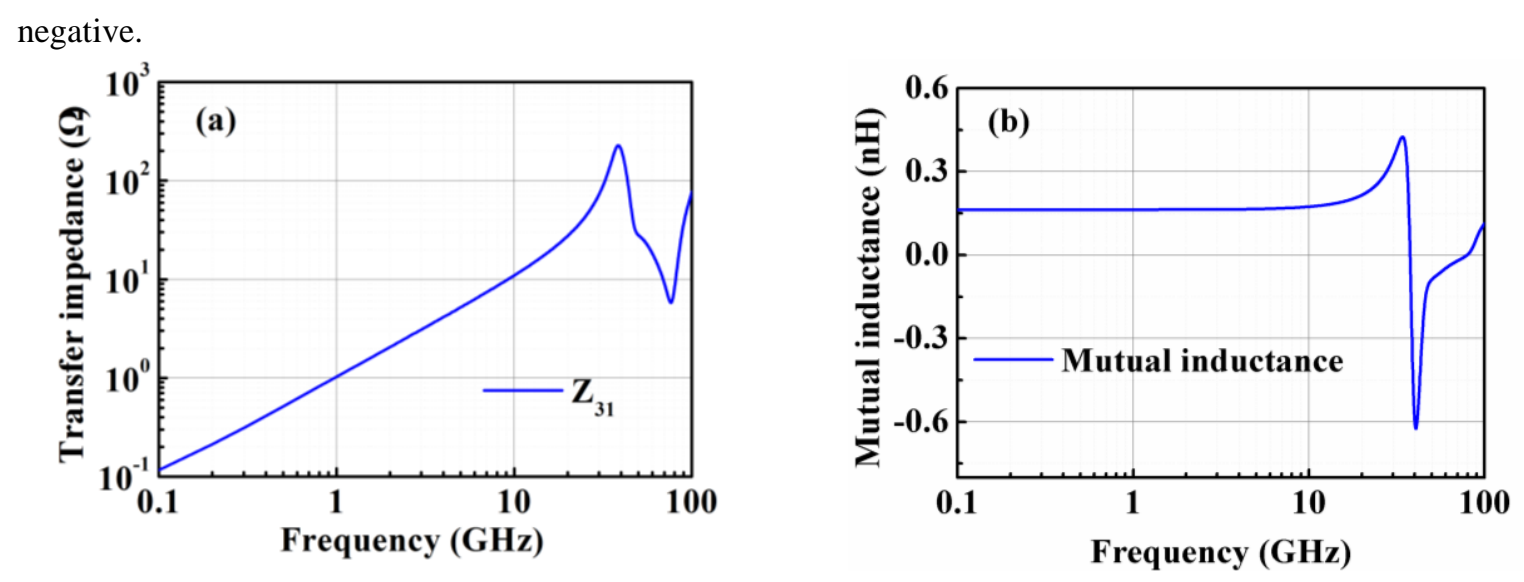

Fig. 3. (a) Transfer impedance between clock channel and CRW and (b) Simulated mutual inductance between Port 3 and Port 1 of the baseline model.

\subsection{Equivalent Circuit Model}

Although the S parameter files obtained from the EM simulation can be directly used as a blackbox in the commercial IC design tools such as Cadence, the complex nature of S parameters due to various parasitic effects makes the time-domain and large-signal simulation like periodic steady state (PSS) or periodic noise (PNoise) very time consuming and even prone to potential convergence problems [20]. Therefore, it is highly desired to build a physics-based lumped element model that can characterize the vertical noise coupling behavior and help in simulating the 3D mixed-signal integrated circuits. From a practical point of view, the equivalent circuit model also needs to be applicable over wide bandwidth, because the sharp rising and falling edge of the clock signal in digital chip contains high frequency components. For instance, to capture the vertical noise coupling generated by a $3 \mathrm{GHz}$ square wave clock signal with 33ps raising time (10\% of signal period), the model should at least cover the $7^{\text {th }}$-order or $9^{\text {th }}$-order harmonic of the fundamental frequency, which means the model should be valid from DC to nearly $30 \mathrm{GHz}$.

Fig. 4(a) shows a basic $\pi$ model that are commonly used for a metal trace routing above the lossy substrate. In this work, two methods are employed to further broaden the bandwidth, first, $2-\pi$ equivalent circuit is adopted which can better described the distributed feature of the long clock channel and CRWs, second, the series R-L in $\pi$ model is replaced by a ladder network consisting of $\mathrm{L}, \mathrm{L}_{\mathrm{f}}, \mathrm{R}_{\mathrm{f}}$ and $\mathrm{R}_{\mathrm{m}}$ in order to capture frequency dependent loss from the metal due to skin effect [20]. As illustrated in Fig. 4(b), the clock channel, CRW 'a' and ' $b$ ' are modeled using three 2- $\pi$ networks separately. The inductive coupling through mutual inductance and capacitive coupling through top substrate among clock channel, CRW ' $a$ ' and ' $b$ ' are then added to the 2- $\pi$ network, while the common ground reference for the whole structure is setting at the top and bottom ground grid connected by multiple TSVs. The physical meaning of each component is explained as follows.

The self-inductances of each CRW and clock channel are represented by series inductance $\mathrm{L}_{\text {signal }}$ and $\mathrm{L}_{\text {clock}}$, respectively, and $\mathrm{M}_{\mathrm{CS}}$ stands for the mutual inductance between them. $\mathrm{C}_{\mathrm{ss}}$ and $\mathrm{M}_{\mathrm{ss}}$ represent the capacitance and mutual inductance between the two parallel CRWs. The capacitance and resistance of top silicon substrate are modeled by $\mathrm{C}_{\text {sub }}$ and $\mathrm{R}_{\text {sub }}$ in parallel, which are determined from the dielectric relaxation time of the substrate [21]. The oxide capacitance between the CRWs and the silicon substrate is modeled by $\mathrm{C}_{\mathrm{IMD} 1}$, and $\mathrm{C}_{\text {bond }}$ is the capacitance between clock channel and top substrate due to the bonding layer. $\mathrm{C}_{\text {signal }}$ and $\mathrm{C}_{\text {clock }}$ model the capacitance from CRWs and clock channel to ground grids, respectively. The values of multiple parameters in the equivalent circuit can be obtained through two steps, first, a set of estimated values are derived directly from analytical calculation [21], which are used as the initial values for parameter fitting and optimization during the second step. Since the variation of the parameters are confined within a limited range around their calculated values based on the physical dimensions and material property, it prevents the iteration from converging to a local minimum or resulting in physically meaningless values. The corresponding parameters of each component are listed in Table II. In order to verify the accuracy of proposed equivalent model, the simulated S parameters from the lumped element model and 3D full-wave EM solver (HFSS) were compared in Fig. 5(a)-(d) over wide bandwidth (i.e. $0.1 \mathrm{GHz}-40 \mathrm{GHz}$ ). It can be seen that, good agreement is achieved for both reflection coefficient ( $\mathrm{S}_{11}$ and $S_{33}$ ) and coupling coefficient $\left(S_{31}\right.$ and $\left.S_{41}\right)$, which proves the broadband nature and accuracy of the proposed model. And the obtained model can be utilized in circuit design software for almost any kinds of simulation without worrying about convergence 
issues.

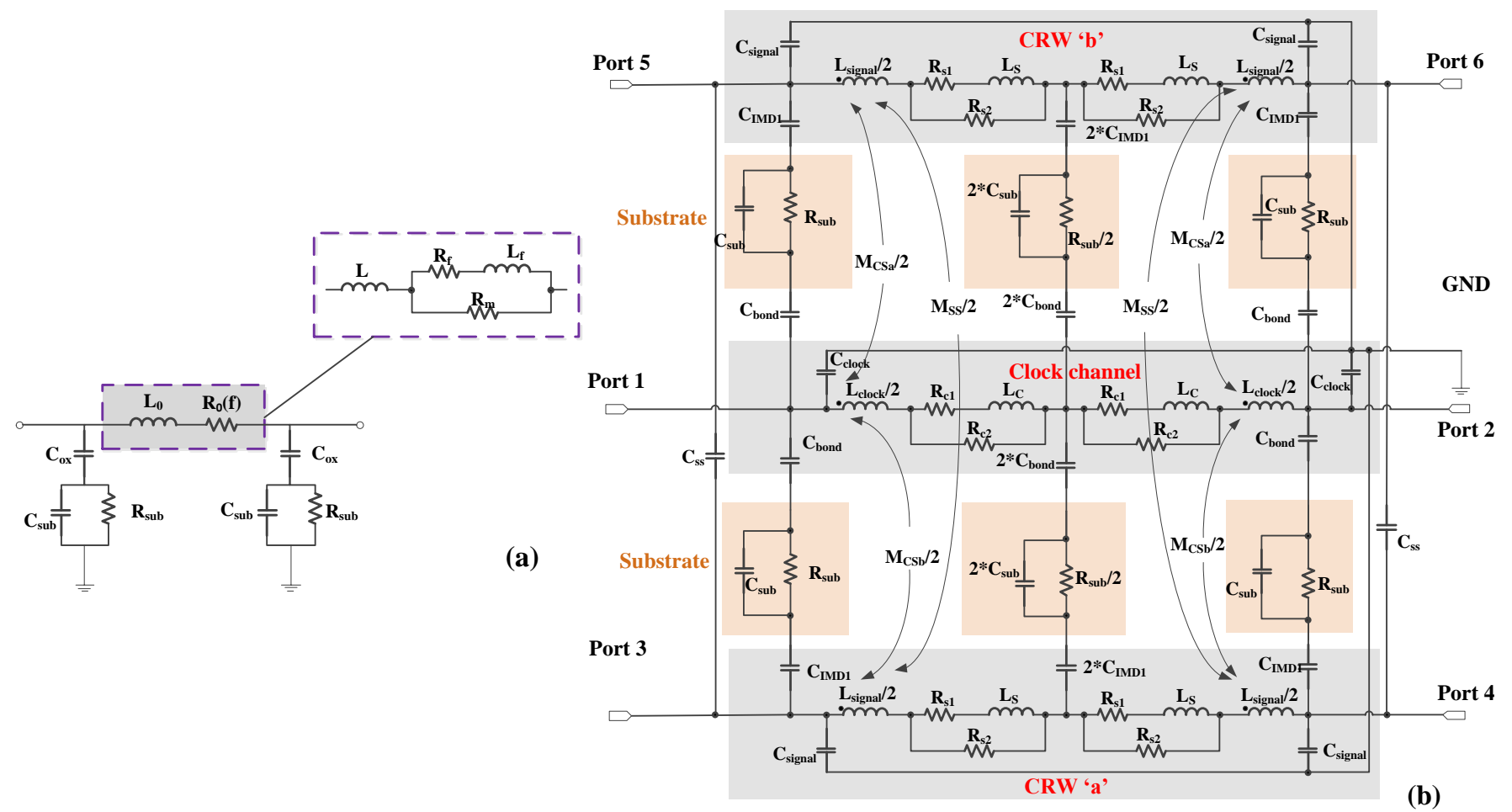

Fig. 4 (a) Basic $\pi$ model for metal trace on lossy substrate; (b) The equivalent circuit model of vertical crosstalk between clock channel and CRWs.
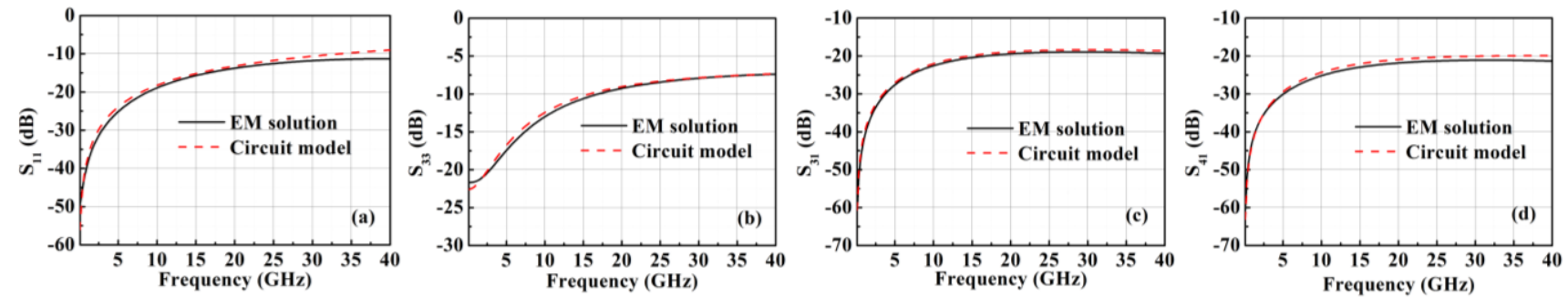

Fig. 5. Comparison of simulated crosstalk between EM solution and equivalent model for (a) $S_{11}$, (b) $S_{33}$, (c) $S_{31}$, (d) $S_{41}$ from $0.1 \mathrm{GHz}$ to $40 \mathrm{GHz}$.

TABLE II

PARAMETERS IN EQUIVALENT CIRCUIT MODEL FROM 0.1GHz TO 40GHZ.

\begin{tabular}{ccccccccc}
\hline \hline $\mathbf{R}_{\mathbf{s 1}}(\mathbf{\Omega})$ & $\mathbf{R}_{\mathbf{s 2}}(\mathbf{\Omega})$ & $\mathbf{R}_{\mathbf{c 1}}(\mathbf{\Omega})$ & $\mathbf{R}_{\mathbf{c 2}}(\boldsymbol{\Omega})$ & $\mathbf{L}_{\mathbf{S}}(\mathbf{p H})$ & $\mathbf{L}_{\mathbf{C}}(\mathbf{p H})$ & $\mathbf{L}_{\text {signal }}(\mathbf{p H})$ & $\mathbf{L}_{\text {clock }}(\mathbf{p H})$ & $\mathbf{C}_{\text {bond }}(\mathbf{f F})$ \\
\hline 4.1 & 166.17 & 0.046 & 53.94 & 37.9 & 89.1 & 442 & 142.4 & 1.96 \\
\hline $\mathbf{C}_{\text {IMD1 }}(\mathbf{f F})$ & $\mathbf{C}_{\mathbf{s s}}(\mathbf{f F})$ & $\mathbf{C}_{\text {signal }}(\mathbf{f F})$ & $\mathbf{C}_{\text {clock }}(\mathbf{f F})$ & $\mathbf{C}_{\text {sub }}(\mathbf{f F})$ & $\mathbf{R}_{\text {sub }}(\mathbf{\Omega})$ & $\mathbf{M}_{\mathbf{C S a}}(\mathbf{p H})$ & $\mathbf{M}_{\mathbf{C S b}}(\mathbf{p H})$ & $\mathbf{M}_{\mathbf{S S}}(\mathbf{p H})$ \\
\hline 30.4 & 12.7 & 2.49 & 15.6 & 8.25 & 1690 & 140 & 140 & 406.64 \\
\hline \hline
\end{tabular}

\section{Impacts of Design Variables on Vertical Noise Coupling}

This section studies the impact of design variables on vertical noise coupling including geometry dimension, different stacking processes, and layout floorplan. Moreover, four methods which can reduce the crosstalk between clock channel and CRWs are discussed and compared.

\subsection{Geometry Dimension}

To investigate the influence of dimension on crosstalk, the length and width of metal traces (i.e. clock channel and CRWs) are varied separately. Fig. 6(a) plots the $S_{31}$ from $0.1 \mathrm{GHz}$ to $100 \mathrm{GHz}$ for different metal length of $600 \mu \mathrm{m}$ and $1200 \mu \mathrm{m}$, and Fig. 6(b) 
depicts the crosstalk simulated at $3 \mathrm{GHz}$ when the metal length varies from $500 \mu \mathrm{m}$ to $1200 \mu \mathrm{m}$. As expected, the crosstalk increases by $5 \mathrm{~dB}$ for both NEXT and FEXT when metal length doubles since the mutual inductance is proportional to metal length. While the FEXT is $2 \mathrm{~dB}$ lower than the NEXT under the same length. Besides, the self-resonance frequency is also decreased for longer metal due to larger self-inductance. Fig. 7 plots the impact of metal width on crosstalk. Since the capacitive coupling is proportional to metal width, while the inductive coupling is a weak function of metal width, and the inductive coupling dominates below $15 \mathrm{GHz}$ as described in section 2.3, the resulting crosstalk is almost unchanged for different metal widths below $15 \mathrm{GHz}$. However, the simulated crosstalk has a observable difference at frequency above $15 \mathrm{GHz}$ because the capacitive coupling starts to manifest itself. As for the impact of TSV parameters, the radius of TSVs as well as the thickness of insulator have almost no impact on crosstalk. However, the depth (length) of TSV which is directly related to the thickness of top substrate does have an impact on vertical noise coupling or crosstalk, because both the inductive coupling and capacitive coupling become stronger as the TSV depth reduces.
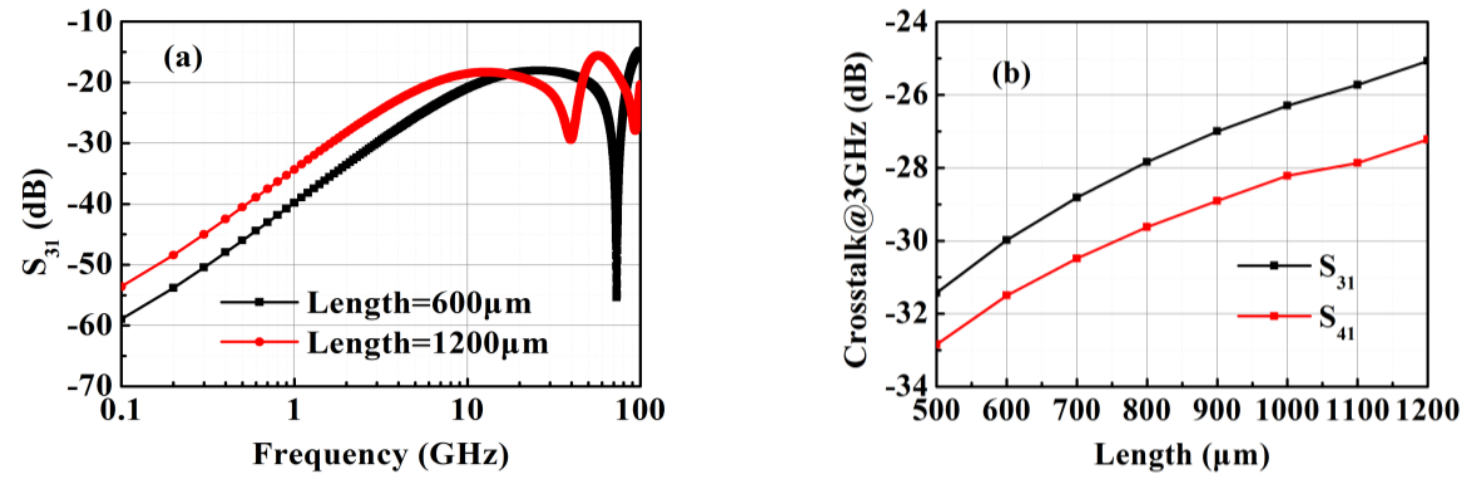

Fig. 6 (a) Comparison of $S_{31}$ with different clock channel and CRW length from $0.1 \mathrm{GHz}$ to $100 \mathrm{GHz}$. (b) Impact of metal length on crosstalk at $3 \mathrm{GHz}$.
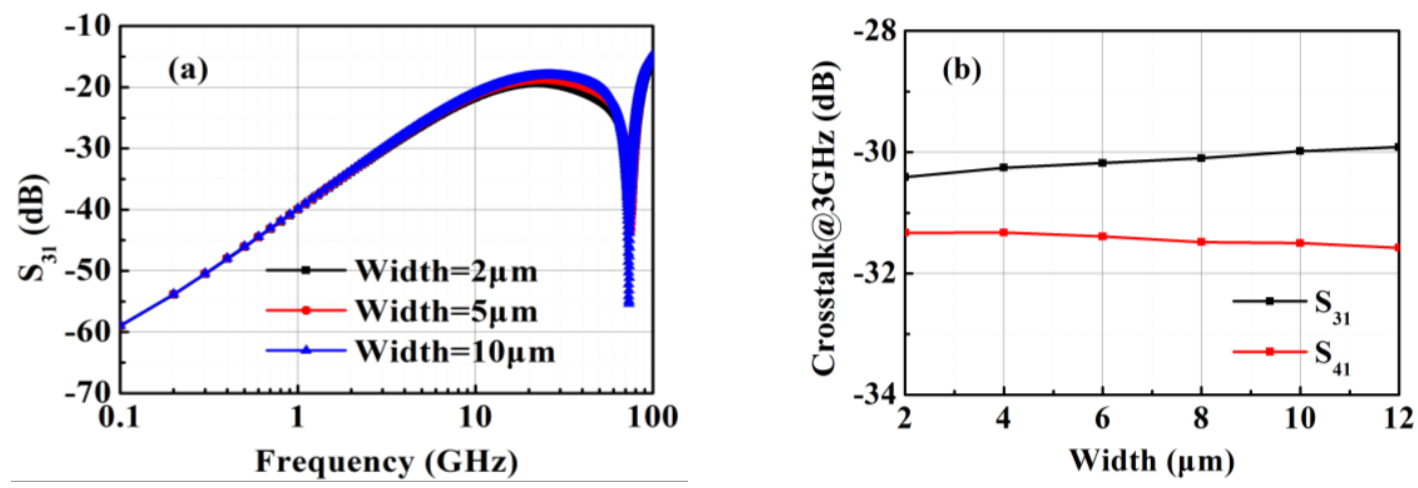

Fig. 7. (a) Comparison of $S_{31}$ with different metal width from $0.1 \mathrm{GHz}$ to $100 \mathrm{GHz}$. (b) Impact of width on crosstalk at $3 \mathrm{GHz}$.

\subsection{D Stacking Process}

The 3D stacking process will also have effect on the crosstalk, such as substrate thickness, face-to-face stacking or face-to-back stacking. Before stacking the analog chip on top of the digital chip, wafer thinning is required to reduce the thickness of top substrate and reveals the bottom of TSVs for micro-bump soldering. The impact of top substrate thickness on crosstalk is illustrated in Fig. 8, where a thinner substrate results in larger crosstalk since shorter coupling path leads to stronger coupling. As we know, the thickness of the substrate is related to TSV depth, which is restricted by the achievable aspect ratio (ratio of depth to diameter) from micro-fabrication process, so there is a trade-off between the crosstalk and substrate thickness or TSV depth. 

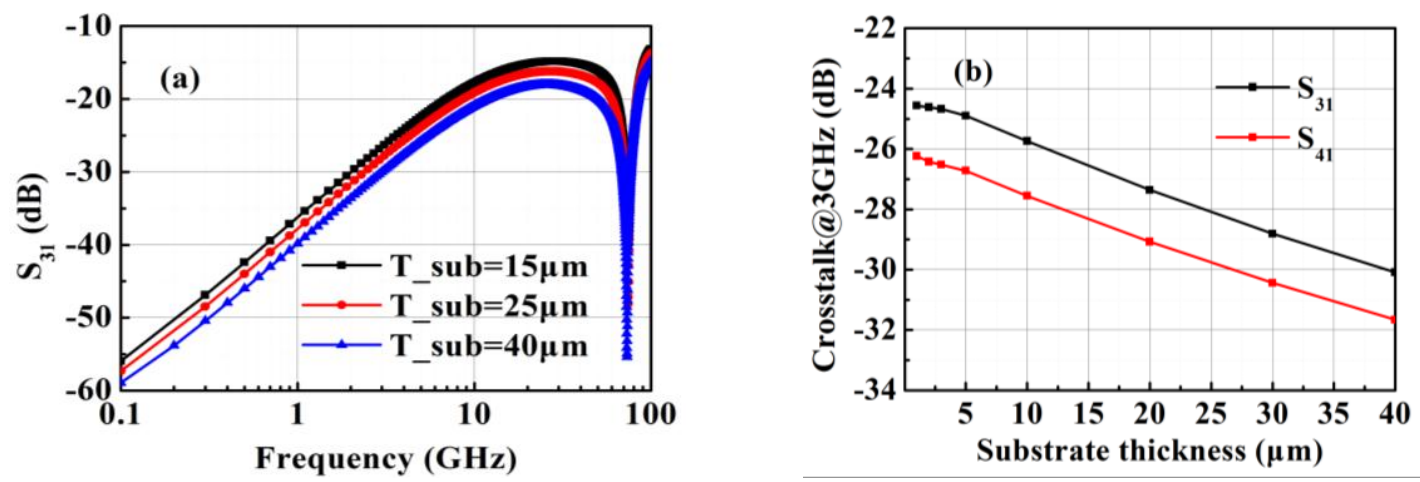

Fig. 8 (a) Comparison of $\mathrm{S}_{31}$ with different thickness of top substrate from $0.1 \mathrm{GHz}$ to $100 \mathrm{GHz}$. (b) Impact of top substrate thickness on crosstalk at $3 \mathrm{GHz}$.

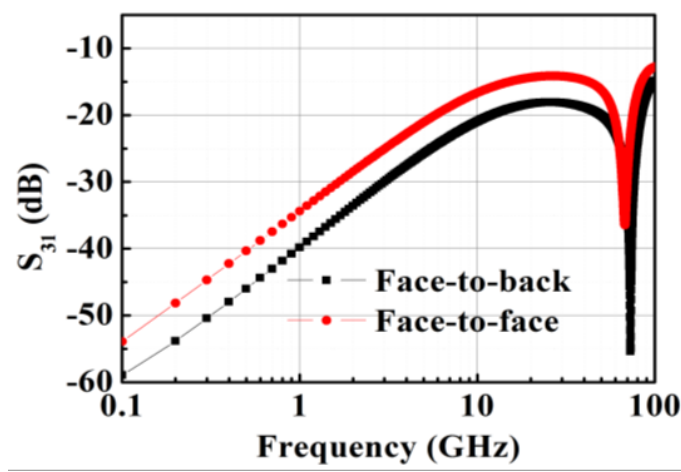

Fig. 9 Comparison of $\mathrm{S}_{31}$ with different bonding process (face-to-face and face-to-back).

In Fig. 9, face-to-face (bottom tier face bonded to top tire face) and face-to-back (bottom tier face bonded to top tire back) bonding processes are compared for crosstalk performance. As expected, coupling noise in face-to-face bonding is worse than face-to-back bonding due to the short distance between clock channel and CRWs.

\subsection{Layout Floorplan}

The baseline model is constructed as the two CRWs located right above the clock channel in parallel with zero offset along $\mathrm{x}$-direction. But in reality, the clock channel may not be in parallel with the CRWs, moreover, even they are in parallel, it is very likely that they offset from each other along the x-direction. Fig. 10 reports the crosstalk from $0.1 \mathrm{GHz}$ to $100 \mathrm{GHz}$ for different wire direction, i.e. clock channel is parallel or perpendicular to CRWs. The result clearly shows the crosstalk in perpendicular case is dramatically decreased compared to the parallel case, because both inductive and capacitive coupling are reduced. Specifically, the inductive coupling is reduced since the magnetic field excited by current flowing through clock channel and CRWs are within two orthogonal planes, and the capacitive coupling is also reduced due to smaller overlap area. Fig.11(a) compares the crosstalk when the horizontal offset $(\Delta \mathrm{x})$ is $0,40 \mu \mathrm{m}$ and $80 \mu \mathrm{m}$. The result indicates that a larger offset leads to a smaller crosstalk, since the distance between clock channel and CRWs is increased. From Fig. 11(b), it is also shown that the offset of two CRWs induces difference between $S_{31}$ and $S_{51}$, as the CRW ' $a$ ' and ' $b$ ' are no longer symmetrical with respect to clock channel. 


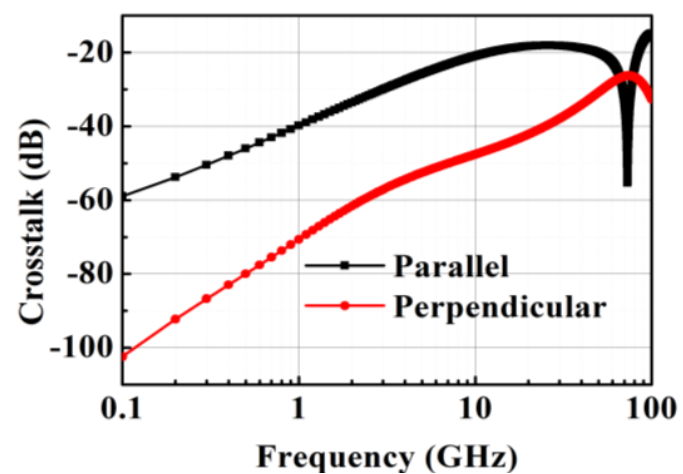

Fig. 10 Comparison of noise transfer functions with different wire direction from $0.1 \mathrm{GHz}$ to $100 \mathrm{GHz}$.
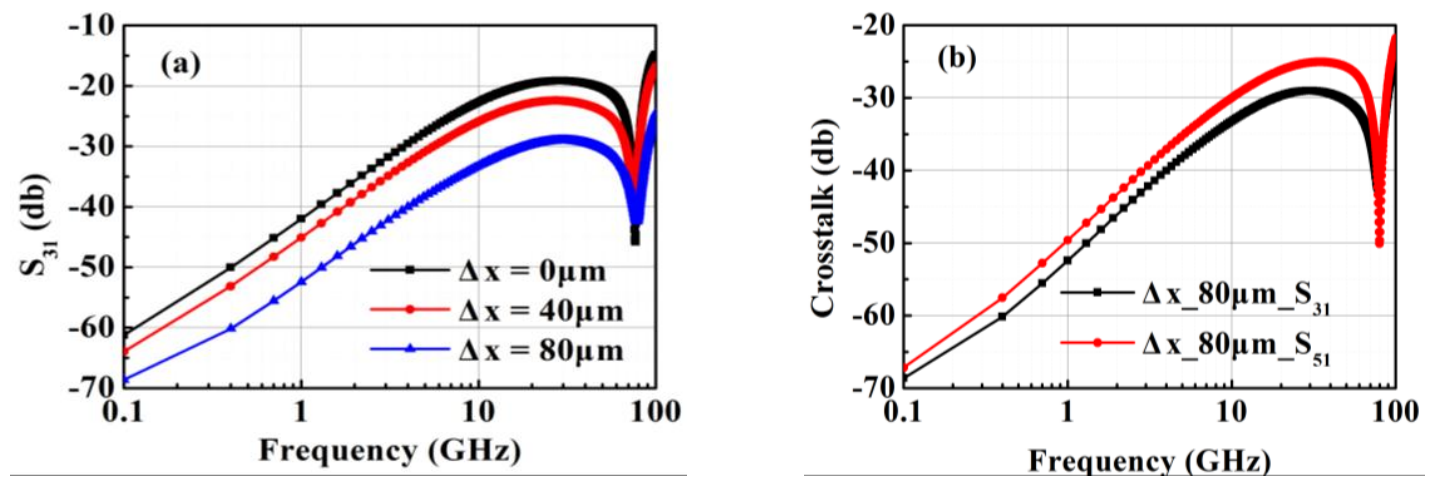

Fig. 11 (a) the comparison of $\mathrm{S}_{31}$ for different offset between clock channel and CRWs; (b) the crosstalk at the port of CRW ' $a$ ' and 'b' under $80 \mu \mathrm{m}$ offset.

\subsection{Methods for Crosstalk Reduction}

This section suggests four possible ways to reduce the vertical noise coupling between the analog and digital chips. The first method is to route the clock channel in perpendicular to the CRWs as discussed in section 3.3, which will greatly reduce the crosstalk. The second way is adding a metal ground plane at the bottom of top substrate to shield the electromagnetic field. As depicted in Fig. 12(a), the value of $S_{31}$ with $1 \mu \mathrm{m}$ thick copper ground plane are $18 \mathrm{~dB}$ lower than the baseline model without ground plane below self-resonant frequency. Another way to isolate the two tiers is to make the ground grids closer to the CRWs, which can reduce the mutual inductance and hence the inductive coupling between clock channel and CRWs. As a comparable study, the space between ground grids and CRWs is reduced from $190 \mu \mathrm{m}$ (baseline model) to $30 \mu \mathrm{m}$ and the simulated $\mathrm{S}_{31}$ is shown in Fig. 12(b) for both cases. The result tells that the crosstalk is much smaller for reduced distance between CRWs and ground grids, which also proves that the inductive coupling is the main coupling mechanism. The last suggested way to improve noise immunity is to make the two CRWs 'a' and 'b' differential so as to cancel the common-mode noise from the digital clock. Fig. 13 compares four different methods for crosstalk reduction, which demonstrates that using differential CRWs is the most effective approach for reducing crosstalk. However, it should be noted that the simulation is based on the ideal case with no horizontal offset between CRWs and clock channel. In other words, the CRW ' $a$ ' and ' $b$ ' are symmetrical with respect to the clock channel, therefore the noise coupling can be cancelled out when subtracting ' $b$ ' from ' $a$ '. And the crosstalk cannot be completely cancelled out if a finite offset exists, as shown in Fig.11(b), which is the most likely scenario in real chip implementation. Besides, using differential CRWs in analog circuits requires more chip area and also consumes more power. 

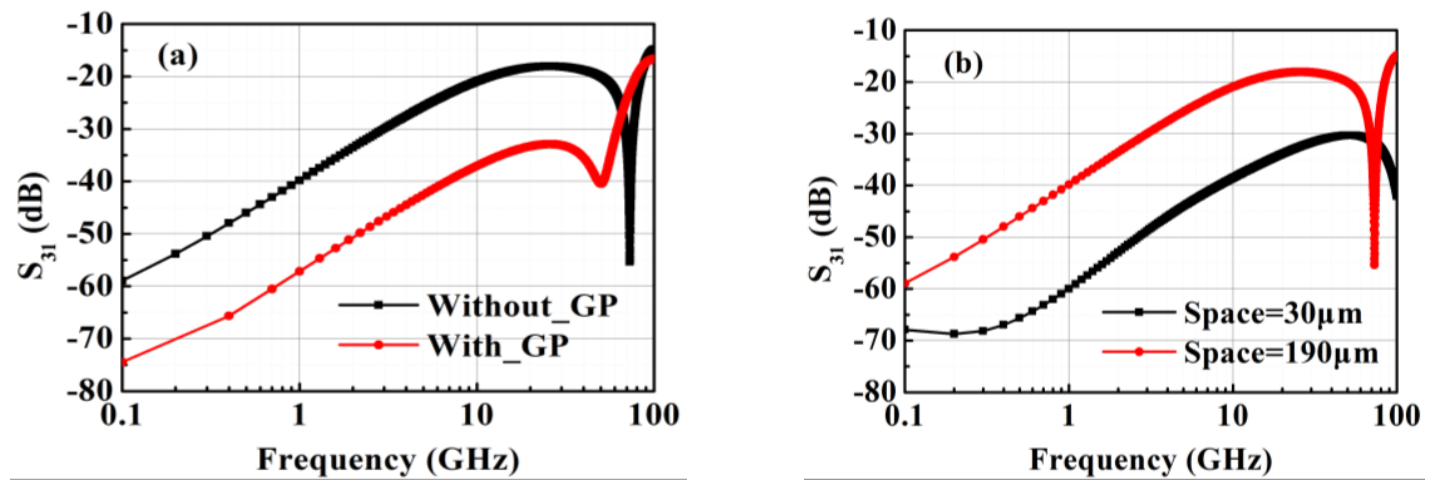

Fig. 12. (a) Comparison of $S_{31}$ with and without ground plane. (b) Comparison of $S_{31}$ for different space between CRWs and ground grids.

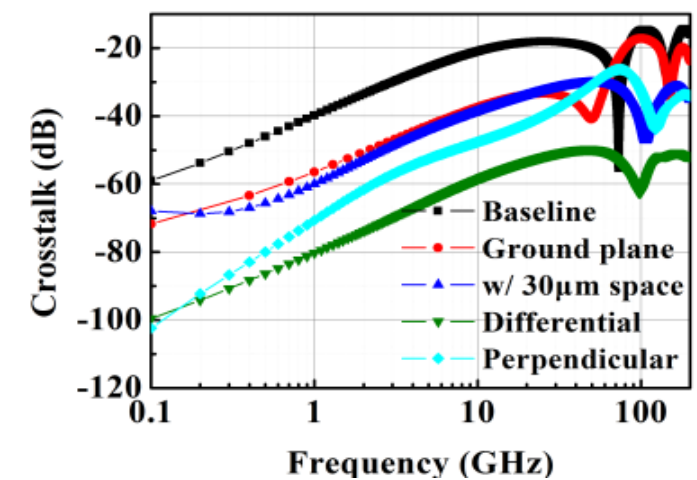

Fig. 13. Comparison of different methods for crosstalk reduction.

\section{Time Domain Analysis}

In this section, to analyze the transient noise coupling characteristics in time domain, the equivalent circuit model in Fig. 4(b) is imported into Advanced Design System (ADS). CMOS inverters are used as drivers and loads at each terminal of the clock channel and CRWs. To ensure the driving capability, the channel length and width of the CMOS inverter driving the clock channel at Port 1 is chosen as $100 \mathrm{~nm}$ and $240 \mu \mathrm{m}$ respectively. While all the other MOSFETs' channel length and width are set to $100 \mathrm{~nm}$ and 5 $\mu \mathrm{m}$. During simulation, the DC bias voltage on CRWs ' $a$ ' and ' $b$ ' is kept zero, and the input of inverter connected to clock channel is driven by a square wave with $1.2 \mathrm{~V}$ amplitude, which is the supply voltage for $90 \mathrm{~nm}$ CMOS technology.

Fig. 14(a)-(f) illustrate the noise voltage measured at the near-end (Port 3) and far-end (Port 4) of CRW when a $1.2 \mathrm{~V}$ clock signal is applied to clock channel with different frequency and rising/falling time. Fig.14(a) plots the transient response to a 0.1 GHz clock signal with $1 \mathrm{~ns}$ rising/falling edge (10\% of clock period), where the NEXT exhibits a $6.8 \mathrm{mV}$ square-shape noise with sharp peaks $\left(11 \mathrm{mV}_{\mathrm{pp}}\right)$ at its rising and falling edge. The square-shape noise is coupled from the $0.1 \mathrm{GHz}$ clock signal, and the peaks are generated by the sharp rising/falling edge of clock signal. Fig.14(b) plots the FEXT with the similar square-shape noise as NEXT but larger peaks $\left(23 \mathrm{mV}_{\mathrm{pp}}\right)$ near the rising/falling edge. This is because the FEXT is transmitting in forward direction and grows larger with longer coupling length [22]. If the rising and falling time is reduced to $100 \mathrm{ps}$ while keeping the same clock period $(0.1 \mathrm{GHz})$, both NEXT and FEXT exhibit ringing due to faster clock switching, and the peak-to-peak noise voltage increases from $11 \mathrm{mV}_{\mathrm{pp}}$ to $52 \mathrm{mV}_{\mathrm{pp}}$ for NEXT and from $23 \mathrm{mV}_{\mathrm{pp}}$ to $173 \mathrm{mV}_{\mathrm{pp}}$ for FEXT, respectively, as shown in Fig. 14(c)-(d). Note that such large noise amplitude will bring nonnegligible influence on analog circuit. When clock frequency is increased to $1 \mathrm{GHz}$ while maintaining the same rising/falling edge (100 ps), the NEXT and FEXT are shown in Fig.14(e)-(f), which also have ringing effect, and the peak-to-peak value of the coupled noise remain the same, since they have same rising/falling time.

To further study the noise transfer characteristics, the Fast Fourier Transformation (FFT) is applied to coupled noise signals in Fig. 14(a)-(f). As depicted in Fig. 15(a)-(b), the noise spectrum clearly shows frequency components at $0.1 \mathrm{GHz}, 0.3 \mathrm{GHz}$ and $0.5 \mathrm{GHz}$, which are the fundamental tone, 3rd and 5th harmonics of the square-shape noise waveform in Fig. 14(a)-(b). However, 
when the rising/falling time reduces from $1 \mathrm{~ns}$ to $100 \mathrm{ps}$, the corresponding spectrum shown in Fig. 15(c)-(d) exhibits more tones besides the harmonics of the clock frequency $(0.1 \mathrm{GHz})$, because the peak-to-peak noise voltage at the rising/falling edge increases dramatically in time domain. Finally, Fig 15(e)-(f) plots the FFT results of the coupled noise from $1 \mathrm{GHz}$ clock with 100 ps rising/falling time, which have frequency components up to around $25 \mathrm{GHz}$. Finally, by comparing the noise spectrum of NEXT with that of FEXT from Fig.15(a)-(f), it can be concluded that the FEXT is more severe than the NEXT, which is consistent with the time domain results shown in Fig.14(a)-(f).
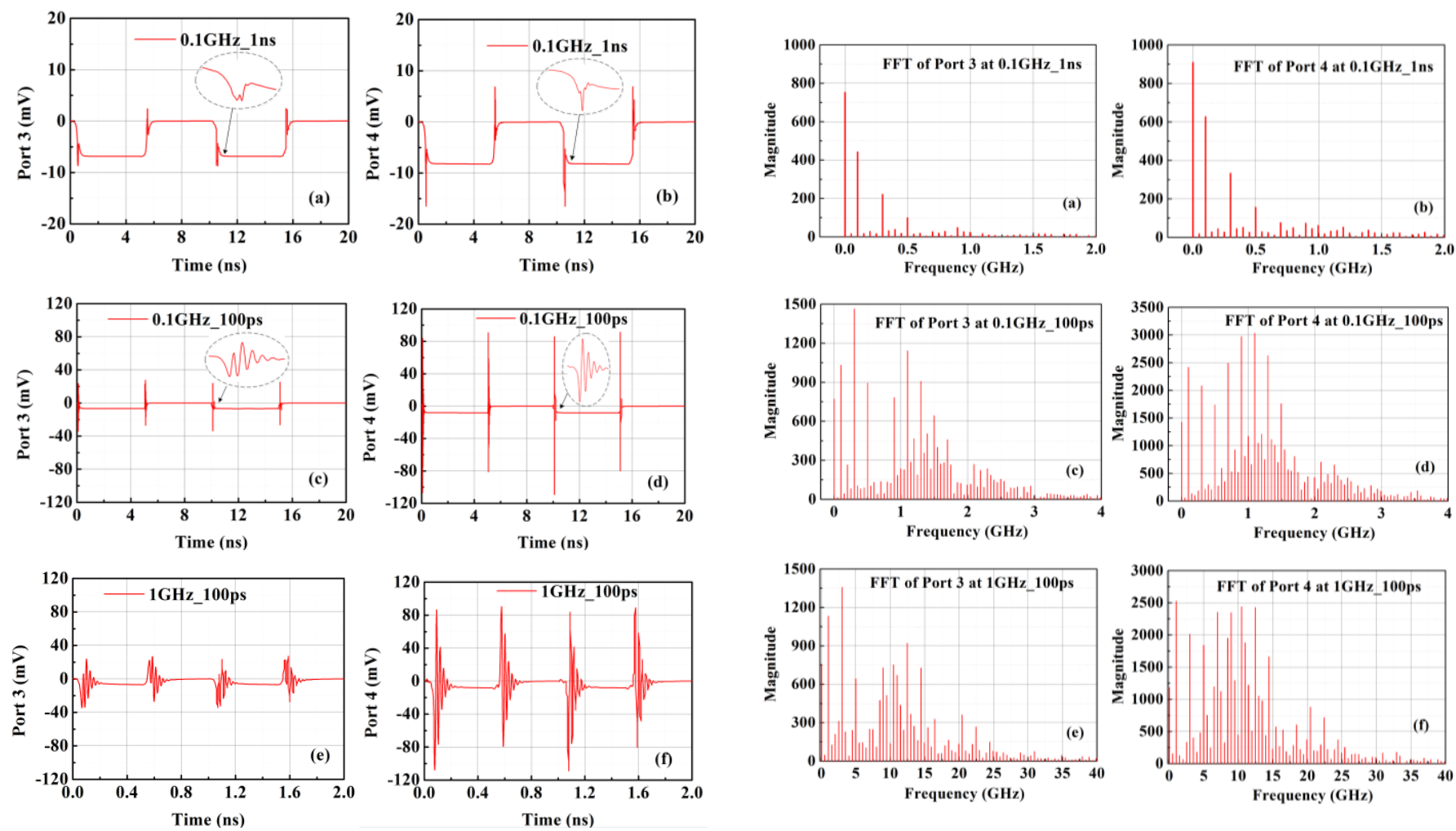

Fig. 14. Simulated NEXT (Port 3) and FEXT (Port 4) of the baseline model in time domain. (a) NEXT from a $0.1 \mathrm{GHz}$ square wave clock signal with $1 \mathrm{~ns}$ rising/falling time, (b) FEXT from a $0.1 \mathrm{GHz}$ square wave clock signal with 1ns rising/falling time, (c) NEXT from a $0.1 \mathrm{GHz}$ square wave clock signal with 100ps rising/falling time, (d) FEXT from a $0.1 \mathrm{GHz}$ square wave clock signal with $100 \mathrm{ps}$ rising/falling time, (e) NEXT from a $1 \mathrm{GHz}$ square wave clock signal with 100ps rising/falling time, (f) FEXT from a $1 \mathrm{GHz}$ square wave clock signal with 100ps rising/falling time.

Fig. 15. Fast Fourier Transformation (FFT) of the noise waveform shown in Fig.14(a)-(f).

\section{Conclusions}

This paper analyzes the vertical noise coupling between clock channel and CRWs in TSV-based 3D mixed-signal integration. Full-wave EM model and broadband equivalent circuit model are built and the simulation results show a good agreement. Coupling mechanism is explored based on transfer impedance, which demonstrates a pure inductive characteristics from $0.1 \mathrm{GHz}$ to $15 \mathrm{GHz}$, while the capacitive coupling manifests itself at frequency above $15 \mathrm{GHz}$. The effect of design variables on the crosstalk is also discussed, such as geometry dimension, different stacking processes, and layout floorplan. Four approaches for improving the noise immunity between clock channel and CRWs are suggested and compared, including keeping CRWs closer to ground grids, clock channel being perpendicular to CRWs, adding a ground plane, and applying differential CRWs. In the end, the noise coupling is simulated in time domain for different clock frequency and rising/falling time, which shows the peak-to-peak noise voltage increases with shorter rising/falling time and the far-end noise magnitude is much greater than the near-end. 
Acknowledgments: This work is supported by National Science Foundation of China under Grant 61574016, 61301006 and 61404008, 111 project of China under Grant B14010, Beijing Institute of Technology Basic Research under Grant 20130542015 and The scholarship from China Scholarship Council (File No. 201306030071).

\section{References}

[1]J. U. Knickerbocker, P. S. Andry, B. Dang, R. R. Horton, M. J. Interrante, C. S. Patel, R. J. Polastre, K. Sakuma, R. Sirdeshmukh, E. J. Sprogis, S. M. Sri-Jayantha, A. M. Stephens, A. W. Topol, C. K. Tsang, B. C. Webb, and S. L. Wright, "Three-dimensional silicon integration," IBM J. Res. Develop., vol. 52, no. 6, pp. 537-664, Nov. 2008.

[2] J.-Q. Lu, “3-D hyperintegration and packaging technologies for micro-nano systems,"Proc. IEEE, vol. 97, no. 1, pp. 18-30, Jan. 2009.

[3]R. S. Patti, "Three-Dimensional Integrated Circuits and the Future of System-on-Chip Designs," Proceedings of the IEEE, vol. 94, no. 6, pp. 1214-1224, 2006.

[4]J. P. Gambino, S. A. Adderly, and J. U. Knickerbocker, “An overview of through-silicon-via technology and manufacturing challenges," Microelectronic Engineering, vol. 135, no. 0, pp. 73-106, 3/5/, 2015.

[5]K. Uksong, C. Hoe-Ju, H. Seongmoo, P. Duk-Ha, L. Hoon, K. Jin Ho, A. Soon-Hong, C. Soo-Ho, A. Jaesung, K. DukMin, L. Jae-Wook, J. Han-Sung, K. Woo-Seop, J. Dong Hyeon, K. Nam Seog, C. Jung-Hwan, C. Tae-Gyeong, Y. Jei-Hwan, C. Joo Sun, K. Changhyun, and J. Young-Hyun, “8 Gb 3-D DDR3 DRAM Using Through-Silicon-Via Technology,” Solid-State Circuits, IEEE Journal of, vol. 45, no. 1, pp. 111-119, 2010.

[6]T. Sekiguchi, K. Ono, A. Kotabe, and Y. Yanagawa, "1-Tbyte/s 1-Gbit DRAM Architecture Using 3-D Interconnect for High-Throughput Computing," Solid-State Circuits, IEEE Journal of, vol. 46, no. 4, pp. 828-837, 2011.

[7]K. Jung-Sik, O. Chi Sung, L. Hocheol, L. Donghyuk, H. Hyong Ryol, H. Sooman, N. Byongwook, M. Joungwook, K. Jin-Guk, P. Hanna, R. Jang-Woo, P. Kiwon, K. Sang Kyu, K. So-Young, K. Hoyoung, B. Jong-Min, C. Hyunyoon, J. Minsoo, H. Cheolmin, L. Jung-Bae, C. Joo Sun, and J. Young-Hyun, “A 1.2 V 12.8 GB/s 2 Gb Mobile Wide-I/O DRAM With 4×128 I/Os Using TSV Based Stacking,” Solid-State Circuits, IEEE Journal of, vol. 47, no. 1, pp. 107-116, 2012.

[8]D. Henry, F. Jacquet, M. Neyret, X. Baillin, T. Enot, V. Lapras, C. Brunet-Manquat, J. Charbonnier, B. Aventurier, and N. Sillon, "Through silicon vias technology for CMOS image sensors packaging." pp. 556-562.

[9]G. Prigozhin, V. Suntharalingam, D. Busacker, R. F. Foster, S. Kissel, B. LaMarr, A. M. Soares, J. Villasenor, and M. Bautz, "Characterization of Three-Dimensional-Integrated Active Pixel Sensor for X-Ray Detection,” Electron Devices, IEEE Transactions on, vol. 56, no. 11, pp. $2602-2611,2009$.

[10]X. Gagnard, and T. Mourier, "Through silicon via: From the CMOS imager sensor wafer level package to the 3D integration," Microelectronic Engineering, vol. 87 , no. 3, pp. 470-476, 3//, 2010.

[11]K. Dae Hyun, K. Athikulwongse, M. Healy, M. Hossain, J. Moongon, I. Khorosh, G. Kumar, L. Young-Joon, D. Lewis, L. Tzu-Wei, L. Chang, S. Panth, M. Pathak, R. Minzhen, S. Guanhao, S. Taigon, W. Dong Hyuk, Z. Xin, K. Joungho, C. Ho, G. Loh, L. Hsien-Hsin, and L. Sung-Kyu, "3D-MAPS: 3D Massively parallel processor with stacked memory." pp. 188-190.

[12] K. W. Lee, H. Hashimoto, M. Onishi, Y. Sato, M. Murugesan, J. C. Bea, T. Fukushima, T. Tanaka, and M. Koyanagi, "A resilient 3-D stacked multicore processor fabricated using die-level 3-D integration and backside TSV technologies." pp. 304-308.

[13] K. Kiyoyama, Y. Ohara, K. W. Lee, Y. Yang, T. Fukushima, T. Tanaka and M. Koyanagi, "A parallel ADC for high-speed CMOS image processing system with 3D structure", in 3D System Integration, 2009. 3DIC 2009. IEEE International Conference on, pp. 1-4, 2009.

[14]W. Liu, G. Chen, X. Han, Y. Wang, Y. Xie and H. Yang, "Design methodologies for 3d mixed signal integrated circuits: A practical 12-bit sar adc design case", in Design Automation Conference (DAC), 2014 51st ACM/EDAC/IEEE, pp. 1-6, 2014.

[15]S. Lee, K. Koo, and J. Kim, “Analysis of die-to-die vertical crosstalk between Clock-tree and Voltage Controlled Oscillator in 3-D IC,” 2011 IEEE International Symposium on Electromagnetic Compatibility (EMC), Aug. 2011, pp.28-32.

[16]K. Koo, M. Kim, J.J Kim, J. Kim, and J. Kim, "Vertical Noise Coupling From On-Chip Switching-Mode Power Supply in a Mixed-Signal Stacked 3-D-IC," IEEE Trans. Compon. Packag. Manuf. Technol., vol.3, no.3, pp. 476-488, March 2013.

[17]E.G. Friedman, "Clock distribution networks in synchronous digital integrated circuits," Proc. of the IEEE, vol.89, no.5, pp. 665-692, May 2001.

[18]N. H. Weste and D. M. Harris, CMOS VLSI design: a circuits and systems perspective: Pearson Education India, 2005.

[19]A. Hastings, The art of analog layout: Prentice Hall, 2006.

[20]Fengyi (Fred) Huang, Jingxue Lu, Nan Jiang, et al. Frequency-Independent Asymmetric Double- $\pi$ Equivalent Circuit for On-Chip Spiral Inductors: Physics-Based Modeling and Parameter Extraction, IEEE JOURNAL OF SOLID-STATE CIRCUITS, 2006, 41(10):2272-2283.

[21]C. P. Yue and S. S. Wong, "Physical modeling of spiral inductors on silicon,” IEEE Trans. Electron Devices, vol. 47, pp. 560-568, Mar. 2000.

[22]E. Bogatin, Signal integrity: simplified: Prentice Hall Professional, 2004. 


\section{Graphical Abstract}
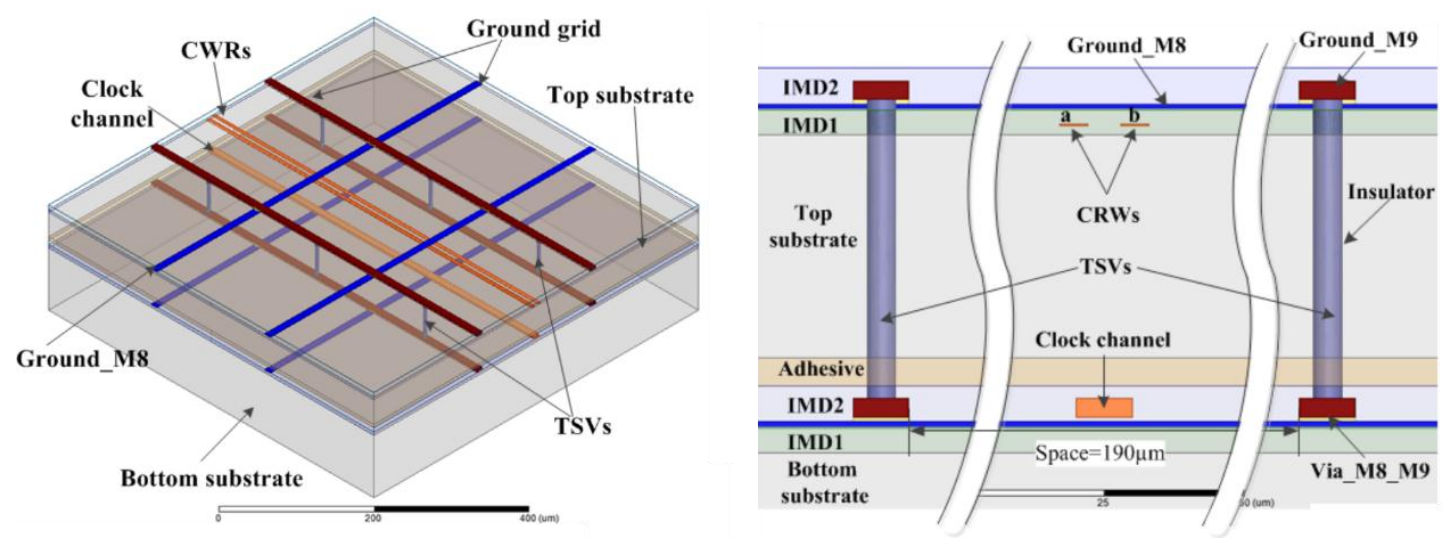